\title{
A New Type of Bio-Chemical Sensor Based on SPM
}

\author{
Huibin Zhao ${ }^{{ }^{*}}$, Quan Ren ${ }^{2}$, Yunsheng Lin $^{1}$, Yansheng Zuo ${ }^{1}$, and Li Han ${ }^{1}$ \\ ${ }^{1}$ Institute of Electrical Engineering, Chinese Academy of Sciences, China \\ ${ }^{2}$ Institute of Mechanics, Chinese Academy of Science, China
}

\begin{abstract}
A new type of Bio-Chemical sensor based on SPM was designed and fabricated in this paper. The Bio-Chemical sensor uses the SPM head as the probe part, which can convert the physical variation quantity on the micro-cantilever to electrical signals, and finally change it into image. In the experiment, the Bio-Chemical sensor based on SPM is used to detect two kinds of ssDNA molecules in PBS solution. By comparing the data in our experiment with that in other experiment, it can be seen that detecting unknown DNA molecules and other chemical or biological molecules in solution by this type of Bio-Chemical sensor is efficient and practical.
\end{abstract}

\section{Keywords-micro-cantilever; bio-chemical sensor; SPM}

\section{INTRODUCTION}

Since the micro-cantilevers were used as force probes in SPM (scanning probe microscope) in 1980's, researchers had began to pay attention to the extreme sensitivity of the micro-cantilevers such as acoustic noise, temperature, humidity, and ambient pressure [1]. In 1994, two research teams, one from Oak Ridge National Laboratory and the other from IBM Zurich, used the micro-cantilevers into a platform for a new family of sensors $[2,3]$. Researcher found that the mass-sensitive of micro-cantilevers outperform more conventional piezoelectric gravimetric sensors (4-6). The sensitivity of micro-cantilevers to minute quantities of adsorbates was superior to that of traditional quartz crystal microbalance (QCM) and surface acoustic wave (SAW) transducers[7]. So the sensors based on micro-cantilevers have very high sensitivity and are wildly used in the field of biological and chemical research[8-12].

The principle of this type sensor is that the sensor can detect optical or electrical changes in deflection or frequency caused by surface stress on functional surface of micro-fabricated cantilever. By measuring shifts in the deflection or resonance frequencies of micro-cantilevers, the researchers were able to know some information of the unknown adsorbate. Researchers can also complete the selection of the chemical or biological molecules which have the character of special adsorption by processing the surface of micro-cantilever.

A new type of Bio-Chemical sensor based on SPM was designed and fabricated in this paper. An experiment was set up to detect two kinds of ssDNA molecules in PBS solution by the Bio-Chemical sensor. By analyzing and comparing the data in our experiment with that in other experiment [13], it can be seen that detecting unknown DNA molecules and other chemical or biological molecules in solution by this type of Bio-Chemical sensor is efficient and practical.

\footnotetext{
*Contacting Author: Huibin Zhao is with Dept.of Micro-nano Fabrication Institute of Electrical Engineering, Chinese Academy of Sciences. (phone:+86 010-62629795; fax +86-010-62627543;

email: zhaohb $(\omega$ mail.iee.ac.cn $)$
}

\section{TECHNIQUES OF EXPERIMENTAL WORK}

\section{A. The Bio-chemical Senser}

Mostly the bio-chemical sensor consists of two parts, as shown in fig. 1. One is the recognition system of molecules; the other is the transition system of physical or chemical signals. First the micro-cantilever acts as a receiver, absorbing the unknown molecules, then acts as a transducer of energy, translating the absorption of molecule to the banding or frequency changing of the micro-cantilever, then the banding or frequency changing will be changed into electrical signal by the transition system.

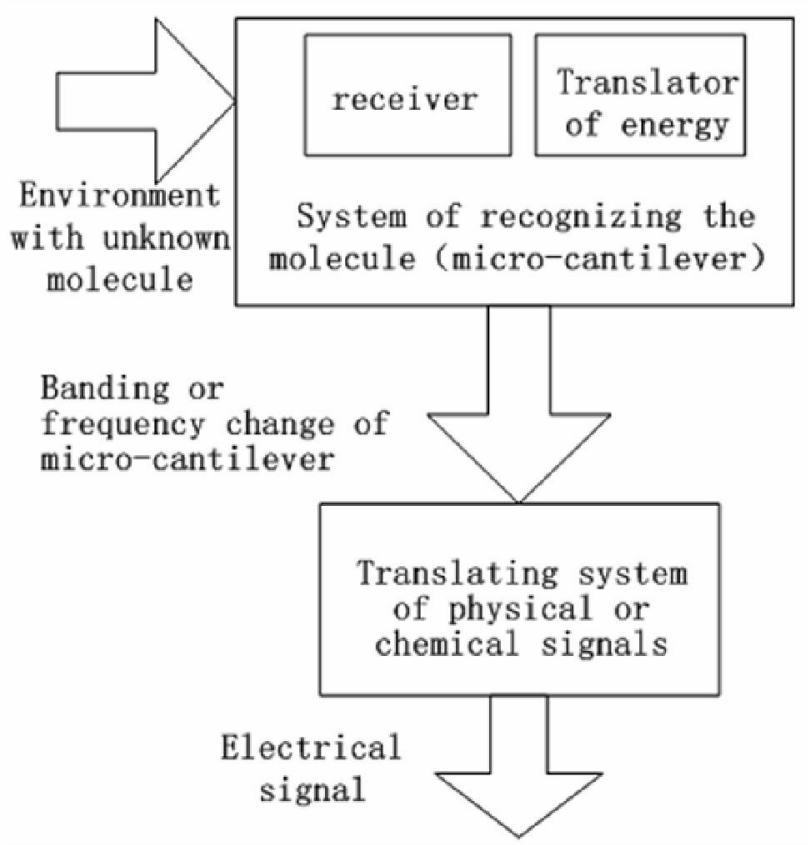

Fig 1. Schematic diagram of a bio-chemical sensor. The micro-cantilever first acts as a receiver, absorbing the unknown molecule, then acts as a translator of energy, translating the absorption of molecule to the banding or frequency changing of the micro-cantilever, then the banding or frequency changing of micro-cantilever will be changed into electrical signal by the translating system.

The sensor was designed based on the SPM-6800 which was developed by our lab. It can detect the surface information of the sample in nanometer scale. When the micro-cantilever with micro-probe is scanning through the sample surface, the interaction between the sample and the probe will influence the micro-cantilever in deflection or resonance frequencies, by measuring shifts in the deflection or resonance frequencies of micro-cantilevers, we can get morphology information of 
sample surface. We use the SPM- 6800 head as the probe of the biochemical sensor. So when the chemical or biological molecules are absorbed on the micro-cantilever surface, the surface compressive stress or the mass of micro-cantilever will be changed, and the deflection or resonance frequencies changes of micro-cantilever will be detected and change into electrical signals by the probe part of the sensor. And then the electrical signals will be changed into digital value and finally changed into image on the screen by the transition system of the sensor. Fig.2 shows the structure of bio-chemical sensor that was design in this paper.

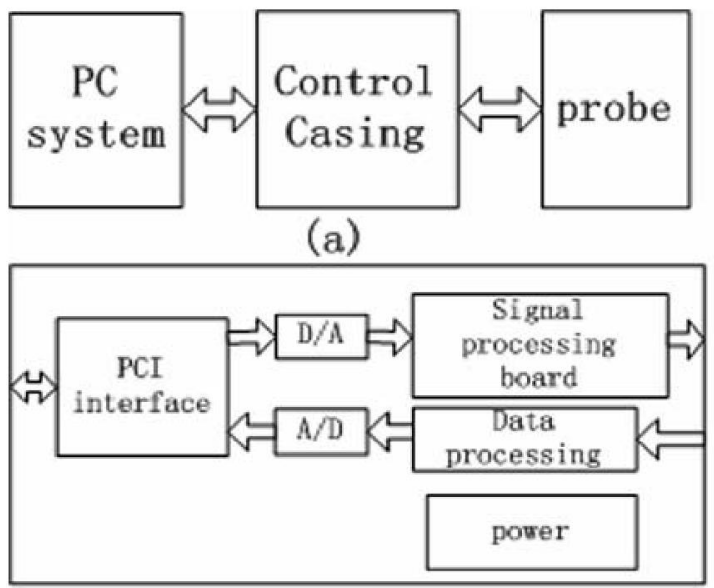

(b)

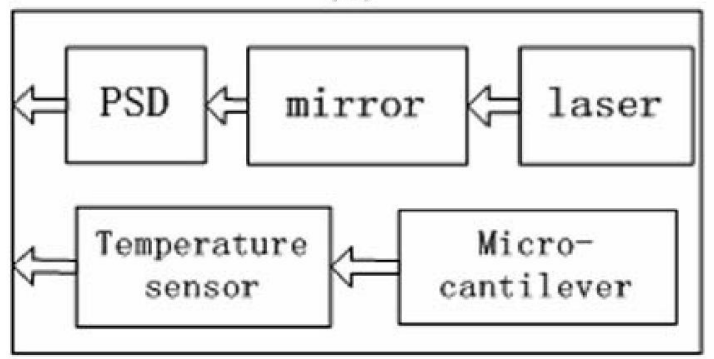

(c)

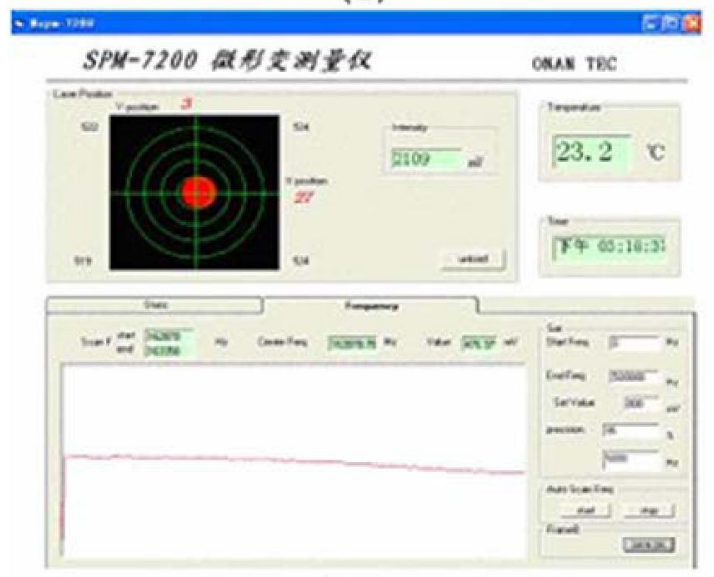

(d)

Fig 2. (a) Structure of whole Bio-Chemical sensor; (b) Structure of control casing; (c) Structure of AFM head; (d) Interface of the software.

\section{B. Experiment Setup}

Generally, during the formation of assembly layer by thiol-modified ssDNA-oligos on a gold surface upon the micro-cantilever, a compressive stress is created, which is the reason of micro-cantilever bent away from adsorbed surface [Fig. 3]. By measuring shifts in the deflection of micro-cantilever, we can know some information of the ssDNA molecules.

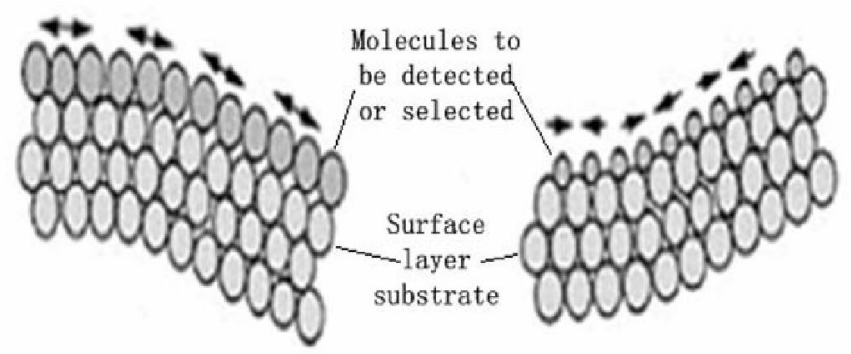

Fig 3. The diagram schematically shows the reason of bending of the micro-cantilever by the surface stress. (a) and (b) show the bending of micro-cantilever by the positive surface stress and the negative surface stress, respectively.

To detect the shifts of chemical adhesion induced by DNA immobility on micro-cantilevers, the optical cantilever reflection technique was used. Fig.4 shows the schematic experimental set-up in this study. When visible light from the low power laser diode is focused on the cantilever apex, the laser beam is reflected off the cantilever surface and collected by a position-sensitive detector (PSD) to transform the currents produced by incident light spot into voltages. And the normalized voltage difference can be digitized to a 16-bit value in an $A / D$ converter, which determines the position of reflected light spot. Then, the changes in deflection can be continuously monitored as well as stored on a computer. In this experiment a liquid cell with syringe pump is necessary because it can control the liquid flow, provide reliable liquid environment, and avoid flow turbulence.

In the experiment, the micro-cantilever was cleaned by the solution which was mix with $\mathrm{H}_{2} \mathrm{O}_{2}(30 \%)$ and $\mathrm{H}_{2} \mathrm{SO}_{4}(98 \%)$ at a volume ratio $3: 7$ before it was put into the fluid cell. This process will remove pollutant on the micro-cantilever surface. Then put the cantilever in the fluid cell with PBS solution. After about $2 \sim 3$ hours, the micro-cantilever reach a stability condition[14] (the deflection is less than $10 \mathrm{~nm}$ per hour), the thiol-modified ssDNA-oligos that was in the PBS solution was slowly injected into the fluid cell by the syringe pump, the PBS solution with ssDNA molecule has same solution concentration as that in the fluid cell[ $[9,15]$. 


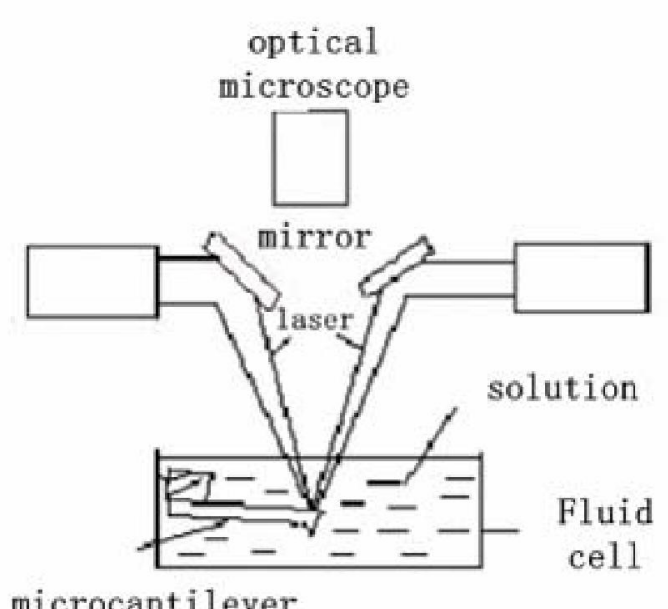

(a)

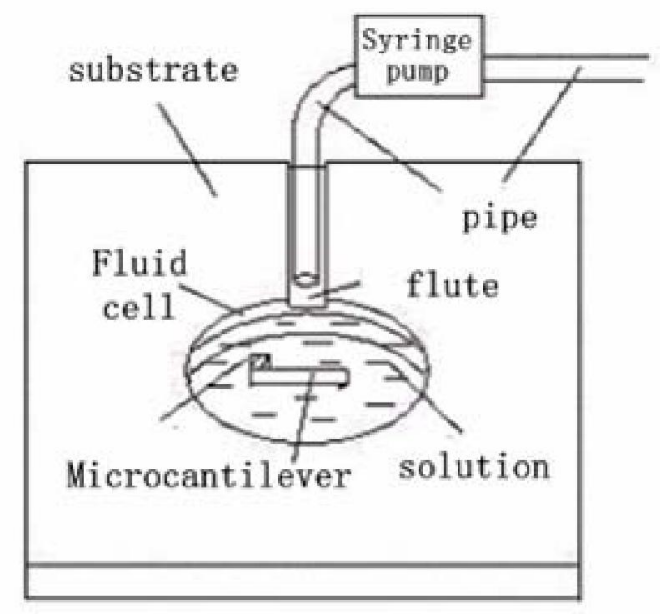

(b)

Fig 4. Schematic of the experimental set-up. (a) The schematic diagram illustrating the instrument (side view). (b) The fluid cell (top view).

the commercial available $\mathrm{V}$-shaped micro-cantilevers, made of silicon nitride $\left(\mathrm{Si}_{3} \mathrm{~N}_{4}\right)$, were used in this experiment, which were $200 \mu \mathrm{m}$ long and $0.5 \mu \mathrm{m}$ thick, and each leg was $20 \mu \mathrm{m}$ wide (NP4, produced by D I company) .One side of cantilever is coated by a thin gold coating serviced to increase the light reflectivity and to immobile the DNA by thiol group.

The lengths of thiol-modified ssDNA used in the experiment were $20 \mathrm{nt}$ and $25 \mathrm{nt}$, and the sequence are S- $\left(\mathrm{CH}_{2}\right)_{6}$-GTA CGA AAT TTG ATT GGC TA and S- $\left(\mathrm{CH}_{2}\right)_{6}$-GTA CGA AAT TTG ATT GGC TAT AAT C, respectively. All the ssDNA solution should be solvent in PBS buffer and stored in $-20^{\circ} \mathrm{C}$.

\section{RESULT AND DISCUSSION}

When the thiol-modified ssDNA-oligos was absorbed on the micro-cantilever surface, the deflection of the cantilever will be monitored and stored on the computer by the bio-chemical senor. In Fig. 5 according to the lower curve that stands for deflection response of micro-cantilever, we can see that the cantilever in the PBS solution had reached a stability condition before $t 1$. We slowly injected the PBS solution with thiol-modified ssDNA-oligos into the fluid cell by the syringe pump. We can see a little turbulence at $t$, it's just the time we injected the PBS solution into the fluid cell. It's shown that the injection had influenced the balance in the fluid cell, and the sensor had detected the little turbulence. After $\Delta t$ the curve stands for deflection response of micro-cantilever goes down and keep on a low level, it means the ssDNA molecules had been absorbed on the cantilever surface, and the cantilever had bent. In Fig. 6 the curves denote the deflection when the ssDNA lengths are $20 \mathrm{nt}$ and $25 \mathrm{nt}$, respectively.

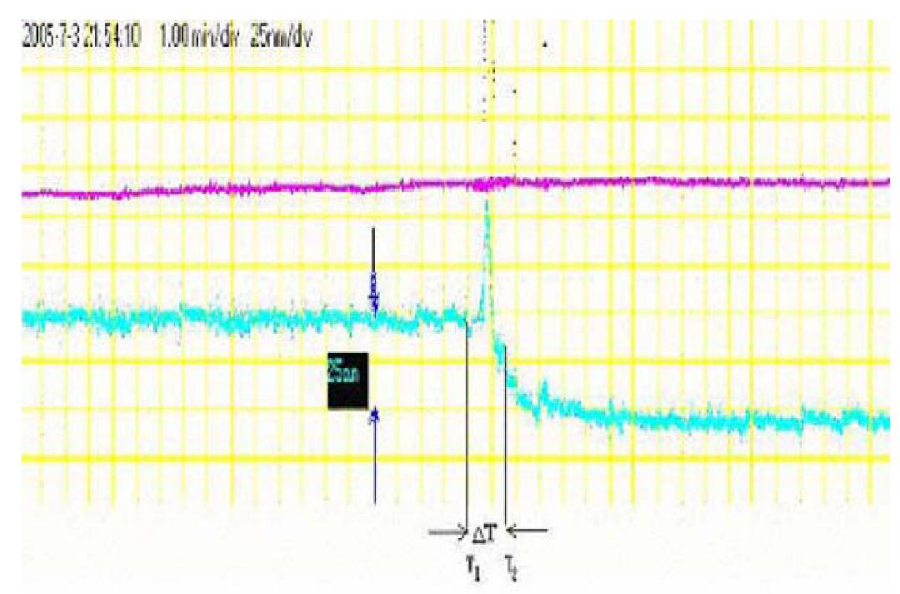

Fig 5. The experimental data directly shown on the computer. The upper curve shows the intensity of the light, and the lower curve is the deflection response when the ssDNA molecules were adsorbed on the microcantilever surface.

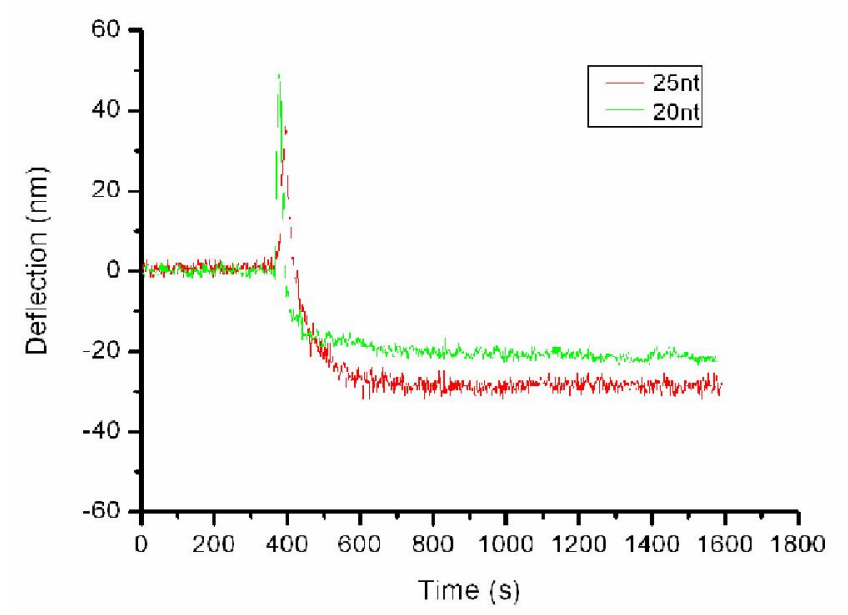

Fig. 6. The deflection response of micro-cantilever versus the time. In this figure the upper curve and the lower curve denote the deflection when the ssDNA lengths are $20 \mathrm{nt}$ and $25 \mathrm{nt}$, respectively. 
According to Stoney's equation [15], Researchers can get some mechanics information of the moleculars which they are interested in.

$$
\Delta \sigma=\frac{E t^{2}}{3 L^{2}(1-v)} \Delta h
$$

where $\Delta \sigma$ is the difference in surface stress between two opposing surface stress, $\Delta z$ is the deflection of the free-end of the cantilever arising from adsorption, $L_{\text {is the length }}{ }^{t}$ is the thickness, $E$ is Young's modulus, and $v$ the Poisson ration of the cantilever.

Comparing the data in our experiment with that in other experiment [13] [Fig. 7], it can be seen that the experiment results are very close. It shows that detecting ssDNA molecules in solution by this type of bio-Chemical sensor is efficient and practical.

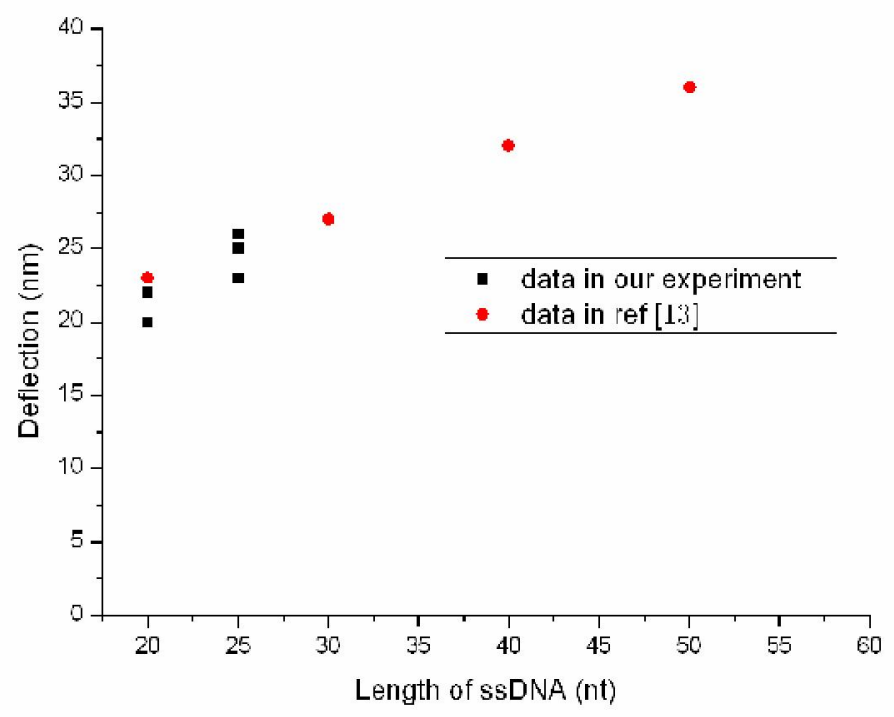

Fig. 7. Changes of the cantilever deflection versus the length of ssDNA. The rectangular points are the data in our experiment, and the circular points are the data by $\mathrm{Wu}[13]$.

\section{CONCLUSION}

A new instrument in the sensor technology based on SPM is designed and fabricated. The sensor can complete the detection or selection of the biological or chemical molecules by the specific adsorption. The experiment result about the biological molecules shows that the sensor is efficient and practical.

The sensor has a good price/performance, now it's only used in research in lab, in the future with the upgrade of the sensor it may detect the quantity or density of molecules in solution, and may be used in the chemical or biological industry.

\section{ACKNOWLEDGMENT}

This work was supported by The National Natural Sciences Foundation of China, granting number: 10427401

\section{REFERENCES}

[1] G. Binning, C. F. Quate and Ch. Gerber, "Atomic Force Microscope" Phys. Rev. Lett, 56 (1986) pp. 930-933.

[2] T. Thundat, R. J. Warmack, G. Y. Chen, and D. P. Allison. "Thermal and ambient-induced deflections of scanning force microscope cantilevers," Appl. Phys. Lett, 64 (1994) pp. 2894-2903

[3] J.R. Barnes, R.J. Stephenson, M.E. Welland, Ch. Gerber and J.K. Gimzewski, "Photothermal spectroscopy with femtojoule sensitivity using a micromechanical device," Nature 372, 1994, pp.79-81.

[4] E. A. Wachter, T. Thundat. "Micromechanical sensors for chemical and physical measurements," Rev. Sci. Instrum. 1995, 66, 3662-3671.

[5] T. Thundat, E. A. Wachter, S. L. Sharp, R. J. Warmack, "Detection of mercury vapor using resonating microcantilevers," Appl. Phys. Lett, 1995, $66,1695-1697$.

[6] T. Thundat, G.Y. Chen, R.J. Warmack, D.P. Allison and E.A. Wachter, "Vapor detection using resonating microcantilevers," Anal. Chem. vol. 67, pp. 519-521, 1995

[7] M Sepaniak, P Datskos, N Lavrik and C Tipple. "Microcantilever Transducers: A New Approach in Sensor Technology," Anal Chem. 2002 Nov 1;74(21):568A-575A.

[8] G Wu, RH Datar, KM Hansen, T Thundat, RJ Cote, A Majumdar. "Bioassay of prostate-specific antigen (PSA) using microcantilevers," Nat. Biotechnol. Biotechnol. 2001, 19, 856-860

[9] J. Fritz, MK. Baller, HP. Lang, H. Rothuizen, P. Vettiger and E.Meyer, "Translating Biomolecular Recognition into Nanomechanics," Science $2000,288,316-318$

[10] P G Datskos, I Sauers. "Detection of 2-mercaptoethanol using gold-coated micromachined cantilevers," Sens. Actuators B, Chem. 1999, 61: $75-82$

[11] K.M. Hansen, H.-F. Ji, G. Wu, R. Datar, R. Cote, et al, "Cantilever-based Optical deflection Assay for the Detection of Single Nucleotide Mismatches," Anal. Chem. 73(7):1567-1571 (2001).

[12] A Boisen, J Thaysen, H Jensenius and O Hansen. "Environmental sensors based on micromachined cantilevers with integrated read-out," Ultramicroscopy, 2000, 82: 11-16

[13] G Wu, H Ji, K Hansen, T Thundat, R Datar, R Cote, et al. "Origin of nanomechanical cantilever motion generated from biomolecular interactions," PNAS, 98 (2001) pp. 1560-1564.

[14] X M Yu, Q H Shang, and X L Jiang. "Bio-Chemical Sensors," PHYSICS, 2002, 31(4): 224-228

[15] G G Stoney. "The tension of metallic films deposited by electrolysis," Proc. R. Soc. London, 1908 Ser, A 82: 172-175 